\title{
Effect of excess oxygen supply on reaction products of a direct ethanol fuel cell
}

\author{
Koichi Niwa, Syohei Hisamatsu, Tomomi Hakuro, Ryousuke Miura, Mutsumi \\ Minakawa and Yasuro Ikuma* \\ Kanagawa Institute of Technology, Atsugi, Kanagawa 243-02, Japan \\ * Corresponding author: e-mail: ikuma@chem.kanagawa-it.ac.jp
}

\begin{abstract}
The effect of various excess oxygen gas flows at the cathode of a direct ethanol fuel cell (DEFC) was investigated with respect to the reaction products/intermediates. The output current increased with the excess oxygen gas flow. For each oxygen flow, the acetic acid concentration at anode was 5 times higher than the acetaldehyde concentration; therefore, acetaldehyde is readily oxidized to acetic acid or acetic acid is formed directly. This suggests that the output current is mainly dependent on the amount of acetic acid produced. The maximum concentration of acetaldehyde with each output current was almost proportional to that of acetic acid for each oxygen flow condition. The effect of cerium oxide addition to the cathode as another oxygen source to enhance performance was also examined. After an excess oxygen gas flow of $0.6 \mathrm{~L} / \mathrm{min}$ was stopped, the output current increased for approximately 2 minutes, after which the cerium oxide re-absorbed oxygen to attain equilibrium, which was accompanied by a decrease in the output current until it returned to the same level as that with the excess oxygen gas flow. Thus, the addition of cerium oxide to the cathode enhances oxygen supply for cathodic reactions with a corresponding increase in output current.
\end{abstract}

Key words: DEFC, Effect of oxygen, Ethanol, Acetaldehyde, Acetic acid

\section{INTRODUCTION}

The difficulties in enhancing electrochemical oxidation during proton generation at the membrane electrode assembly (MEA) of a direct ethanol fuel cell (DEFC) have been discussed in several publications ${ }^{1-3)}$. The amount of intermediates or products such as acetaldehyde or acetic acid is an important parameter to determine the primary chemical reaction taking place in the process of proton generation.

The oxidation rate of intermediates will have a certain limit under the given conditions, such as the flow rate of ethanol aqueous solution, electrode area and concentration of the fuel solution. The output current of a DEFC will be produced as a result of proton and electron chemical reactions that occur at both the anode and cathode. Most research on DEFCs has focused mainly on the oxidation of ethanol and intermediates at the anode. The major aim of such research is to improve the chemical reactions at the anode without consideration for the reactions that occur at the cathode. As such, there has been little research that considers the improvement of reactions at the cathode.

To the best of our knowledge there is only one report $\left.{ }^{4}\right)$ in the literature in which excess oxygen was supplied to the cathode of DEFC. However, in that report, detailed study of excess oxygen was not performed. In this work, we apply a small excess oxygen gas flow to the cathode and evaluate the effect on the amount of intermediate (acetaldehyde). The effect of excess oxygen on the output current of the DEFC is also evaluated and discussed based on the chemical reactions at both the cathode and anode. The output currents of DEFCs and also direct methanol fuel cells (DMFCs) are dependent on the chemical reactions at both the anode and cathode, i.e., the production of intermediates at the anode will be affected by the chemical reactions at the cathode. Furthermore we modify the cathode by mixing Pt/carbon catalyst with $\mathrm{CeO}_{2}$, because we think that it will act as an oxygen supply.

\section{PURPOSE}

Chemical reactions $\mathrm{A}$ and $\mathrm{B}$, shown as equations (1-4), are examples of possible reactions that occur at the anode and cathode during fuel cell operation. Protons and electrons produced at the anode will react with oxygen at the cathode to produce water, which will require sufficient oxygen to consume chemicals at both electrodes. These chemical reactions must occur on both electrodes to achieve the appropriate output current; therefore, if there is insufficient oxygen, then a poor output current will result. As such, first purpose of the present study was to investigate the effect of excess oxygen supply to the cathode on the performance of DEFC. We attempted to supply an excess oxygen flow toward the cathode to evaluate how oxygen directly affects the output current and the formation of intermediates at the anode.

$$
\begin{aligned}
& \mathrm{A} \mathrm{C}_{2} \mathrm{H}_{5} \mathrm{OH} \rightarrow \mathrm{CH}_{3} \mathrm{CHO}+2 \mathrm{H}^{+}+2 \mathrm{e}^{-} \quad \text { Anode (1) } \\
& 1 / 2 \mathrm{O}_{2}+2 \mathrm{H}^{+}+2 \mathrm{e}^{-} \rightarrow \mathrm{H}_{2} \mathrm{O} \quad \text { Cathode } \\
& \mathrm{B} \mathrm{CH} 3 \mathrm{CHO}+\mathrm{H}_{2} \mathrm{O} \rightarrow 2 \mathrm{H}^{+}+2 \mathrm{e}^{-}+\mathrm{CH}_{3} \mathrm{COOH} \text { Anode (3) } \\
& 1 / 2 \mathrm{O}_{2}+2 \mathrm{H}^{+}+2 \mathrm{e}^{-} \rightarrow \mathrm{H}_{2} \mathrm{O} \quad \text { Cathode (4) }
\end{aligned}
$$


In addition, we attempted to control the gas atmosphere at the cathode without the use of excess oxygen gas flow, but by adding suitable additives to the cathode paste Cerium oxide was selected as a cathode additive to control the oxygen partial pressure at the cathode, which should improve the chemical reaction at the anode. The effect of cerium oxide addition to the cathode on the output current is also of significant interest. It is expected that the cathode will become sensitive to the oxygen partial pressure by the addition of cerium oxide. Cerium oxide has the formula $\mathrm{CeO}_{(2-\mathrm{x})}$, where $0<\mathrm{x}<0.28$. It has been reported that $\mathrm{x}$ is dependent on both the temperature and oxygen partial pressure. Ceria releases oxygen to result in an anion deficient non-stoichiometric form that retains the fluorite lattice at high temperatures. This can be described by the following defect reaction, written in Kröger-Vink notation ${ }^{5)}$ :

$\mathrm{Oo}^{\mathrm{x}}+2 \mathrm{CeCe}^{\mathrm{x}} \rightarrow \mathrm{Vo}^{\cdots}+2 \mathrm{CeCe}^{\prime}+1 / 2 \mathrm{O}_{2}(\mathrm{~g})$

where $\mathrm{Oo}^{\mathrm{x}}$ and $\mathrm{VO}_{\mathrm{O}} \cdots$ are oxygen ions in the lattice and doubly charged oxygen vacancies, respectively, which are balanced with $\mathrm{Ce}^{3+}$ or $\mathrm{CeCe}^{2}$. The second purpose of the present study was to find the effect of cerium oxide addition to the cathode on the performance of DEFC.

\section{EXPERIMENTAL}

The anode was prepared by adding $\mathrm{SiO}_{2}$ nanoparticles (10 wt. \%) to a Pt-Ru/carbon catalyst powder (90 wt. \%). $\mathrm{SiO}_{2}$ was purchased from Aerosil, Japan and was in the form of particles with $7 \mathrm{~nm}$ in diameter. Pt-Ru/carbon catalyst powder (TEC61E54DM) was purchased from Tanaka Kikinzoku Kogyo, Japan and was a mixture of 29.5 wt. $\% \mathrm{Pt}+22.9$ wt. $\% \mathrm{Ru}$ and carbon. In the $\mathrm{SiO}_{2}$ mixing process, acetone was added as an additional solvent for uniform dispersion of the nano silica powder ${ }^{6)}$. After ball milling was completed, acetone was removed by vacuum distillation to obtain a paste with a suitable viscosity. The paste of $0.10 \mathrm{~g}$ was painted onto carbon paper $(28 \times 28 \mathrm{~mm})$ to form the anode electrode. The cathode electrode $(28 \times 28 \mathrm{~mm})$ was prepared with $\mathrm{Pt} /$ carbon catalyst powder (TEC10E70TPM) which was purchased from Tanaka Kikinzoku Kogyo and was a mixture of 66.7 wt. \% Pt and carbon. In some cases, $\mathrm{CeO}_{2}$ particles were mixed into $\mathrm{Pt} /$ carbon catalyst at 10 wt. $\%$. The anode, Nafion film, and the cathode were put together and hot-pressed at $200^{\circ} \mathrm{C}$ to form MEA (Fig. 1).

The ceria powder had nanoparticle size but was agglomerated and the purity was $99 \%$. Before mixing with the Pt/carbon catalyst powder, the agglomerated powder was classified into powders with particle sizes under $700 \mathrm{~nm}$ (Fig. 2). In this study we mixed 10 wt. \% $\mathrm{CeO}_{2}$ in cathode only because 10 wt. $\% \mathrm{SiO}_{2}$ was mixed into anode. In the future work, the amount of ceria powder should be considered as an important parameter to enhance the output current of DEFC and should be optimized.

The fuel cells used in this experiment were made of two cells that were electrically connected in series. Fuel was supplied to the inlet of a central common tank located between the two cells. The cathodes (air electrodes) were outside of both cells and were exposed to air. A Perista $\AA$ pump was used to circulate $3 \%$ ethanol aqueous solution of $250-300 \mathrm{~mL}$ at a flow rate of ca. $2 \mathrm{~mL} / \mathrm{min}$. The amounts of acetaldehyde and acetic acid produced during the proton generation process were determined using gas chromatography and $\mathrm{pH}$ measurements. $\mathrm{CO}_{2}$ is one of products that could be found in ethanol solution when ethanol is oxidized. However, it was reported in the literature ${ }^{7)}$ that $\mathrm{CO}_{2}$ was not essential products. We also have found that the concentration of $\mathrm{CO}_{2}$ in ethanol solution did not increase very much after the fuel cell operation. Consequently, in this study we did not measure the concentration of $\mathrm{CO}_{2}$.

The output currents of the DEFCs were measured by the method shown elesewhere ${ }^{4,8}$ ) (resistance of $10 \Omega$ was inserted in series to the circuit for electricity measurements). Every $30 \mathrm{~min}$ of operation, approximately $2 \mathrm{~mL}$ of fuel was sampled to determine the concentration of acetaldehyde and acetic acid generated in the ethanol aqueous solution. Although the cathodes were exposed to air, excess oxygen gas was supplied from a gas cylinder through two funnels set at the cathode side of the DEFC at several different oxygen gas flow conditions: $0,0.2$, and $0.6 \mathrm{~L} / \mathrm{min}$.

During the $2 \mathrm{~mL}$ sampling process, continuous circulation of ethanol aqueous solution was maintained and an attempt was made to avoid air bubbles coming into the fuel aqueous solution. A schematic illustration of the experimental equipment is shown in Fig. 1. Although only one cell is shown in this figure, two cells were used with a central common fuel tank. In this study, the cells were operated at room temperature.

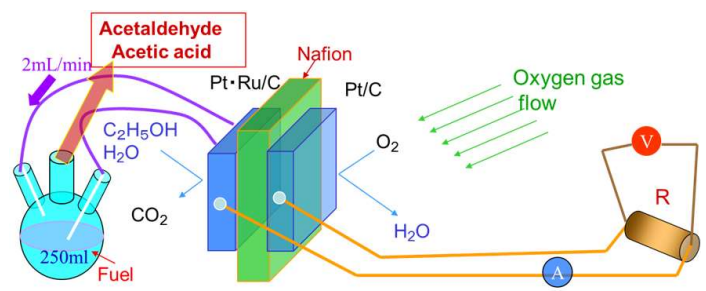

Fig. 1. Schematic illustration of the experimental equipment.

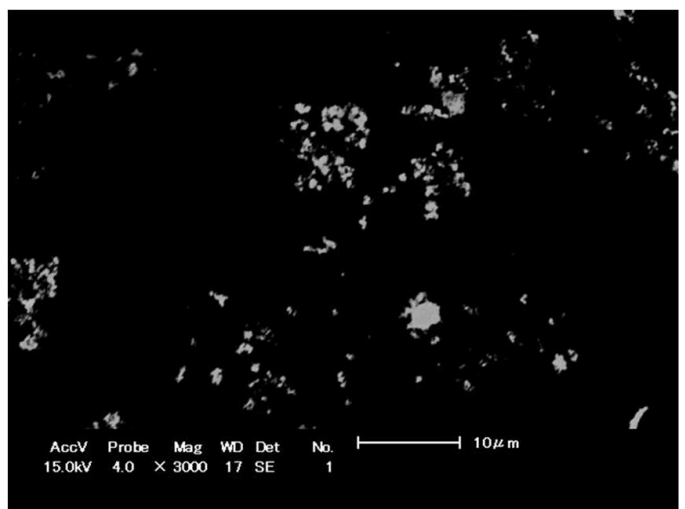

Fig. 2. Classified cerium oxide powder used in this experiment.

\section{RESULTS AND DISCUSSION}

The output current of the DEFC was measured while 
changing the oxygen gas flow rate at a load resistance of $10 \Omega$. A higher oxygen gas flow rate gave a higher output current, as shown in Fig. 3. The current of the DEFC increased rapidly within a period of ca. 20 minutes after the measurement was started. However, the current became almost flat, after which there was a slight decrease in the output current for both oxygen gas flow rates of $0 \mathrm{~L} / \mathrm{min}$ and $0.2 \mathrm{~L} / \mathrm{min}$. However, at an oxygen gas flow of $0.6 \mathrm{~L} / \mathrm{min}$ there was a slight increase in the current.

A large amount of acetaldehyde intermediate was observed for an excess oxygen gas flow rate of $0.6 \mathrm{~L} / \mathrm{min}$ (Fig. 4). However, when the excess oxygen gas flow rate was changed to $0.2 \mathrm{~L} / \mathrm{min}$, the fuel cell did not produce a larger amount of acetaldehyde than the normal $0 \mathrm{~L} / \mathrm{min}$ oxygen gas flow condition after 60 min of operation time. The amount of acetaldehyde is considered to be an important indicator of alcohol oxidation at the anode. This result indicates that a large amount of protons $\left(\mathrm{H}^{+}\right)$ is produced at the anode when ethanol is mostly oxidized into acetaldehyde. If this is true, then Fig. 4 would not explain the results in Fig. 3. The amount of acetaldehyde should be increased as a result of enhanced ethanol oxidation rate at the anode by the supply of excess oxygen gas flow at the cathode. However, Fig. 4 shows that when $0.2 \mathrm{~L} / \mathrm{min}$ of excess oxygen was supplied, the amount of acetaldehyde was not larger than that at $0 \mathrm{~L} /$ min oxygen gas flow. The output currents of both excess oxygen gas flow cases showed that the production of acetaldehyde may not be essential to improve the output current. In our previous work ${ }^{6}$, in which $\mathrm{SiO}_{2}$ powders were mixed with several different particle sizes into the anode of the DEFC, a large amount of acetaldehyde was not essential to realize a high output current. The DEFC in which the silica powder mixed into the anode had a particle size as small as $7 \mathrm{~nm}$ showed the highest output current, but the amount of acetaldehyde produced was the smallest ${ }^{8)}$.

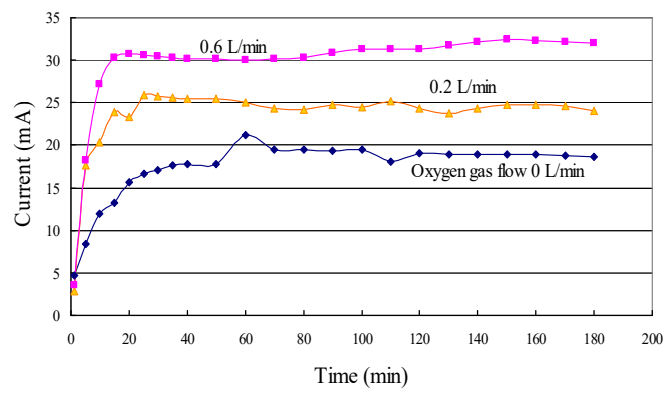

Fig. 3. Output current as a function of the fuel cell operation time under excess oxygen flow.

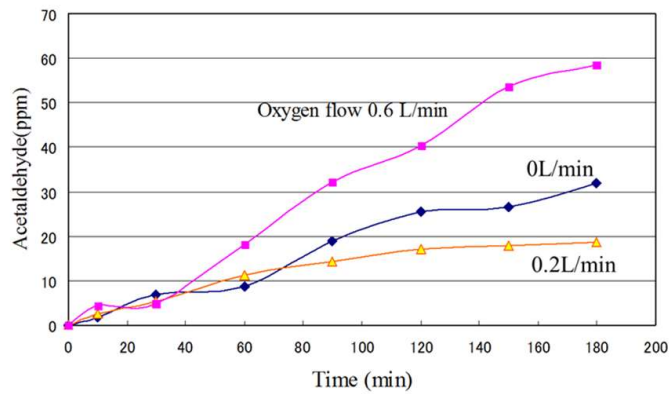

Fig. 4. Amount of acetaldehyde during fuel cell operation for several excess oxygen gas flows.

The relationship between the amount of acetic acid produced and the operation time was almost linear for 90 min (Fig. 5). When $0.6 \mathrm{~L} / \mathrm{min}$ of excess oxygen gas flow was applied to the cathode, an amount of acetic acid larger than that at $0.2 \mathrm{~L} / \mathrm{min}$ excess oxygen gas flow was produced. The concentration of acetic acid was 5 times higher than that of acetaldehyde, which suggests that most of the acetaldehyde at the anode tends to change to acetic acid immediately or ethanol changes directly into acetic acid with unknown reason. The possibility that the presence of $\mathrm{SnO}_{2}$ may oxidize ethanol molecules directly to acetic acid was reported previously ${ }^{9}$. However, most of the research does not indicate the possibility of a direct change from ethanol to acetic acid, but instead indicate the presence of several other intermediates, such as acetaldehyde or $\mathrm{CH}_{3} \mathrm{CO}$ acetyl groups ${ }^{10,11)}$. If a high output current is produced only by the reaction to produce acetaldehyde, then the change in the amount of acetaldehyde due to operation of the fuel cell at $0.2 \mathrm{~L} / \mathrm{min}$ oxygen gas flow (Fig. 4) should produce a smaller output current. However, this prediction does not agree with the result shown in Fig. 3.

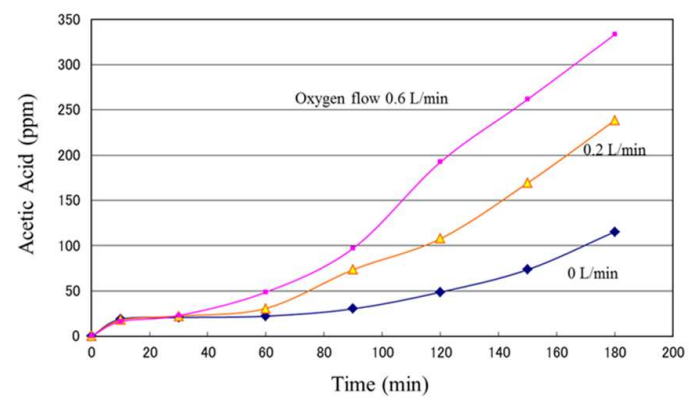

Fig. 5. Amount of acetic acid produced during fuel cell operation.

In Figures 3, 4 and 5, it seems that the trend in the current (rapidly increased within a period of $0-20 \mathrm{~min}$ ) is different from the trend in the amounts of acetaldehyde and acetic acid (gradually increased). This is probably caused by the fact that fuel was circulated at a flow rate of $2 \mathrm{~mL} / \mathrm{min}$ and returned to flask $(250-300 \mathrm{~mL})$. The returning fuel was mixed with original fuel in flask where 
the acetaldehyde and acetic acid were diluted. Then the small amount of fuel was sampled from the flask to measure the concentration. Consequently, the concentrations of acetaldehyde and acetic acid during the first 100-150 min were low.

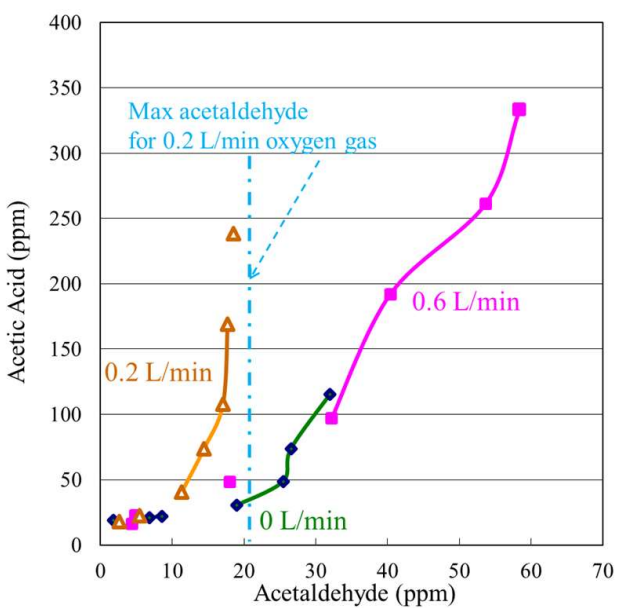

Fig. 6. Relationship between acetic acid and acetaldehyde at several excess oxygen gas flows.

The amounts of both acetaldehyde and acetic acid have a direct or indirect relation to the output currents, as shown in Figures 4 and 5. Combining Figs. 4 and 5, the relationship between acetaldehyde and acetic acid when various excess oxygen gas flow rates were applied is obtained. The results are shown in Fig. 6. For excess oxygen gas flow rates of $0.6 \mathrm{~L} / \mathrm{min}$ and $0 \mathrm{~L} / \mathrm{min}$, a larger amount of acetaldehyde gave a larger amount of acetic acid, but at $0.2 \mathrm{~L} / \mathrm{min}$, there was a maximum amount of acetaldehyde (the amount of acetaldehyde was saturated), even though the amount of acetic acid had increased. This may suggest that the output current is not dependent on the amount of acetaldehyde, but rather is dependent on the amount of acetic acid. An increase in acetaldehyde would be understood to indicate no oxidation process had occurred after the first oxidation process, which is ethanol aqueous solution to acetaldehyde for an excess oxygen gas flow of $0.2 \mathrm{~L} / \mathrm{min}$, i.e., this causes an increase in justaccumulated acetaldehyde.

Combining Figs. 3, 4 and 5, the relationship between concentration of acetic acid and acetaldehyde and the output current is obtained. The results are shown in Fig. 7. The peaks in the amount of acetic acid and acetaldehyde were increased as the flow rate of the applied oxygen gas was increased. These peaks of acetaldehyde almost coincide with the peaks of acetic acid for each oxygen flow condition. The high concentrations of these intermediates were obtained by a high flow rate of oxygen gas applied at the cathode. However, the highest current within the same excess oxygen gas flow was obtained when the concentrations decreased to a level lower than that of the peak value (Fig. 7).

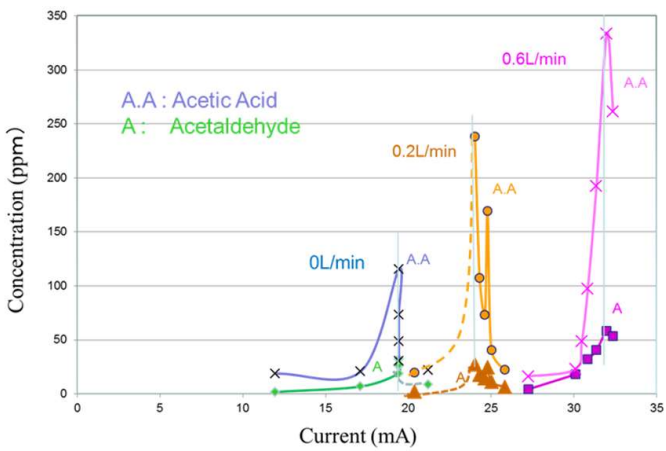

Fig. 7. Both acetic acid and acetaldehyde concentrations as a function of the DEFC output current for several excess oxygen flows.

Figure 8 shows the behavior of cerium oxide in the cathode, when the oxygen supply $(0.6 \mathrm{~L} / \mathrm{min})$ was stopped at ca. $89 \mathrm{~min}$ of fuel cell operation. DEFCs that do not include cerium oxide show a simple decrease in the output current. DEFCs with cerium oxide show an increase in the output current for ca. 2 min. The output current then decreased to the level of the first stage, which indicates that the output current level was that with an excess oxygen gas flow of $0.6 \mathrm{~L} / \mathrm{min}$. Another output current versus working time curve in Fig. 8 shows similar increase in the output current for ca. $2 \mathrm{~min}$. However, it shows slightly smaller output current. Since the chemical reaction in cathode electrodes depends on dispersion condition of cerium oxide, the difference in output currents among these results is an experimental error. The error in output current measurement was $\pm 3 \mathrm{~mA}$. The small decrease in output current at 95 min was caused by the formation of air bubbles in the circulating fuel aqueous solution as we sometimes noticed the air bubbles during the experiment. The fact that the output current returned to $15 \mathrm{~mA}$ level at $96 \mathrm{~min}$ supports this explanation.

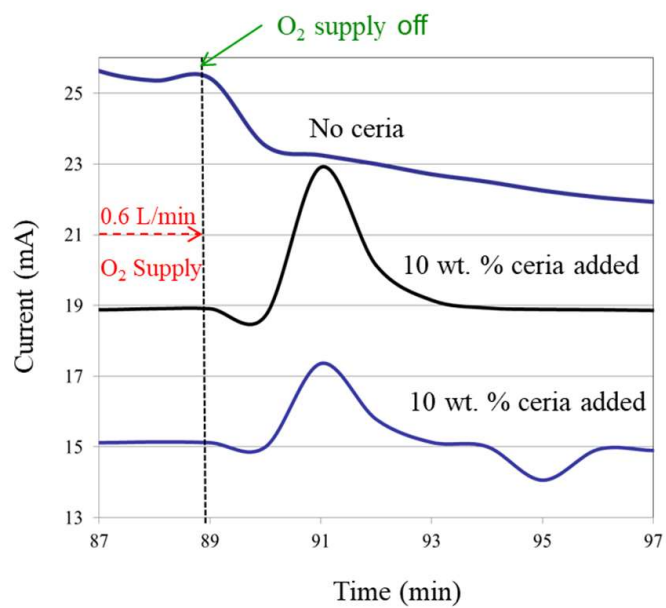

Fig. 8. DEFC output currents with/without cerium oxide in the cathode. 
The increase in output current for ca. 2 min may be explained in the following way. If the supply of excess oxygen gas flow to the cathode is stopped after a period of oxygen supply, then non-equilibrium conditions may be realized at the cathode, which enhances the supply of oxygen from the cerium oxide lattice. Chemical reaction at the cathode would then be enhanced and this would introduce an increase of the output current within a short period of time. The cathode with cerium oxide showed smaller current than that without cerium oxide, because the existence of cerium oxide could hinder the exposure of $\mathrm{Pt}$ to fresh oxygen. The addition of $\mathrm{CeO}_{2}$ accelerates oxygen supply for the cathode without any external oxygen supply (apart from ambient air) even at temperatures as low as room temperature. High concentration of $\mathrm{Ce}_{\mathrm{Ce}}$ in nanocrystalline $\mathrm{CeO}_{2}$ near room temperature was reported in the literature. ${ }^{12)}$

\section{SUMMARY}

A small amount of oxygen gas flow was supplied to the cathode of a DEFC, and the effect of the oxygen gas flow on the amounts of intermediates such as acetaldehyde and acetic acid was evaluated. A cathode mixed with cerium oxide was also prepared, which enhanced the oxygen atmosphere and resulted in enhanced chemical reactions at both electrodes. The results are summarized as follows. - The concentration of acetic acid was 5 times higher than that of acetaldehyde for every excess oxygen gas flow, which suggests that the most of the acetaldehyde at the anode immediately became acetic acid or acetic acid is produced directly from ethanol with known reason.

- The previous result suggests that the output current is not mainly dependent on the amount of acetaldehyde, but rather on the amount of acetic acid produced.

- The formation of intermediates at the anode was affected directly by changing the chemical reactions at the cathode. This is because chemical reactions at the anode and the cathode are interconnected.

- In the plot of concentration versus current (Fig. 7), the current that gives the maximum concentration of acetaldehyde was almost the same as that for acetic acid for each oxygen flow condition: $0,0.2$ and $0.6 \mathrm{~L} / \mathrm{min}$.

- For DEFCs in which cerium oxide was mixed into the cathode, non-equilibrium conditions were established when the oxygen supply $(0.6 \mathrm{~L} / \mathrm{min})$ was stopped at ca. $89 \mathrm{~min}$ of fuel cell operation, and the output current was increased for ca. 2 min. This result suggests that the addition of cerium oxide enhances the chemical reactions at the cathode as well as the anode, which resulted in an increase in the output current.

\section{REFERENCES}

1) J. Mann, N. Yao, and A. B. Bocarsly, Langmuir, 22, 10432-10436 (2006).

2) P. Barecuzuk, A. Lewera, K. Miecznikowski, P. Kuleszuka, and J. Augustynsk, Electrochemical and Solid-State Letters, 12 (12) B165-B166 (2004).

3) J. M. S. Ayoub, R. F. B. De Souza, J. C. M. Silva, R. M. Piasentin, E. V. Spinace, M. C. Santos, and A. O. Neta, Int. J. Electrochem. Sci., 7, 11251-11362 (2012).

4) K. Niwa, S. Hisamatsu, Y. Ikuma, Trans. Mat. Res. Soc. Japan, 41 [2] 175-178 (2016).

5) F. A. Kroger, H. J. Vink, Solid State Phys., 3:307-435
(1956).

6) K. Niwa, T. Tanaka, Y. Ikuma, Key Engineering Materials, 617, 85-88 (2014).

7) J.-M. Jin, T. Sheng, X. Lin, R. Kavanagh, P. Hamer, P. $\mathrm{Hu}, \mathrm{C}$. Hardacre, A. Martinez-Bonastre, J. Sharman, D. Thompsett, and W.-F. Lin, Phys. Chem. Chem. Phys., 16 , 9432-9440 (2014).

8) K. Niwa, R. Murata, K. Arai, Y. Ikuma, Trans Mat. Res. Soc. Japan. 39, 43-46 (2014).

9) R. M. Antoniassi, A. Olivieira Neto, M. Linardi, E. V. Spinace, Int. Journal of Hydrogen Energy, 38, 1206912077 (2013).

10) H. F. Wang, Z. P. Liu, J. Am. Chem. Soc., 130, 10996-11004 (2008).

11) I. Kim, O. H. Hon, S. A. Chen, Y. Park, S. Kwon, K. Lee, Y. Sung, H. Kum, Angew. Chem. Int. Ed., 50, 22702274 (2011).

12) A. Tschope, J. Y. Ying, K. Amonlirdviman, and M. L. Trudeau, Mat. Res. Soc. Symp. Proc., 351, 251256(1994)

(Received March 3, 2018; Accepted November 20, 2018; Published Online February 1, 2019) 